\title{
Changes in cell-surface carbohydrates of Trypanosoma cruzi during metacyclogenesis under chemically defined conditions
}

\author{
Arnaldo F. B. de Andrade, ${ }^{1}$ Manuel J. G. Esteves, ${ }^{1}$ Jayme Angluster, ${ }^{1}$ \\ Magaly Gonzales-Perdomo ${ }^{2,3}$ and Samuel GoldenberG ${ }^{3 *}$ \\ ${ }^{1}$ Instituto de Microbiologia, UFRJ, Cidade Universitaria, Rio de Janeiro, Brazil \\ ${ }^{2}$ Universidad Central de Venezuela, Venezuela \\ ${ }^{3}$ Fiocruz, Dept. Bioquimica e Biologia Molecular, Avenida Brasil 4365, 21040 Rio de Janeiro RJ, Brazil
}

(Received 29 April 1991; revised 6 August 1991; accepted 5 September 1991)

\begin{abstract}
Highly purified lectins with specificities for receptor molecules containing sialic acid, $\boldsymbol{N}$-acetylglucosamine (DGlcNAc), $\boldsymbol{N}$-acetylgalactosamine (D-GalNAc), galactose (D-Gal), mannose-like residues (D-Man) or L-fucose (LFuc), were used to determine changes in cell-surface carbohydrates of the protozoal parasite Trypanosoma cruzi during metacyclogenesis under chemically defined conditions. Of the D-GalNAc-binding lectins, BS-I selectively agglutinated metacyclic trypomastigotes, MPL was selective for replicating epimastigotes, whereas SBA strongly agglutinated all developmental stages of $T$. cruzi. WGA (sialic acid and/or D-GlcNAc specific) was also reactive with differentiating epimastigotes and metacyclic trypomastigotes but displayed a higher reactivity with replicating epimastigote forms. A progressive decrease in agglutinating activity was observed for jacaline (specific for D-Gal) during the metacyclogenesis process; conversely, a progressive increase in affinity was observed for RCA-I (D-Gal-specific), although the reactivity of other D-Gal-specific lectins (PNA and AxP) was strong at all developmental stages. All developmental stages of $T$. cruzi were agglutinated by Con $A$ and Lens culinaris lectins (specific for D-Man-like residues); however, they were unreactive with the L-fucose-binding lectins from Lotus tetragonolobos and Ulex europaeus. These agglutination assays were further confirmed by binding studies using ${ }^{125}$ I-labelled lectins. Neuraminidase activity was detected in supernatants of cell-free differentiation medium using the PNA hemagglutination test with human A erythrocytes. The most pronounced differences in lectin agglutination activity were observed between replicating and differentiating epimastigotes, suggesting that changes in the composition of accessible cell-surface carbohydrates precede the morphological transformation of epimastigotes into metacyclic trypomastigotes.
\end{abstract}

\section{Introduction}

During its life cycle the protozoal parasite Trypanosoma cruzi alternates between different morphological and functional types and different hosts, namely mammals and triatomine (cone-nosed) bugs. The infective and non-replicative trypomastigotes result from differentiation of replicative epimastigotes (within the invertebrate host) and of amastigotes (within mammal cells) (Brener, 1973).

Transformation of epimastigotes into metacyclic trypomastigotes (metacyclogenesis) will also occur in vitro under chemically defined conditions (Contreras et al.,

Abbreviations: LIT, liver infusion tryptose medium; TAU(P), artificial triatomine urine medium (+ proline); Ep-TAU(P), epimastigotes from TAU(P); Ep-LIT, epimastigotes from LIT; Mt, metacyclic trypomastigotes.
1985; Bonaldo et al., 1988). In addition to morphological changes, the metacyclogenesis process involves differential gene expression (Contreras et al., 1985; Bonaldo et al., 1988) and changes in fatty acid composition (Esteves et al., 1989). The process also involves changes in surface carbohydrates, since epimastigotes and trypomastigotes display different lectin agglutination profiles (Pereira $e t$ al., 1980).

The study of changes in surface components during differentiation is of the utmost importance in view of the interaction of the parasite with its intermediary hosts and its adaptive responses to different environments. In this article we analyse changes in the lectin agglutination profile during metacyclogenesis of $T$. cruzi under chemically defined conditions. The results indicate that important changes occur in the pattern of surfaceexposed carbohydrates and that these changes precede 
the morphological transformation of epimastigotes into metacyclic trypomastigotes.

\section{Methods}

Parasites. T. cruzi clone Dm28c, isolated from opossum (Contreras $e t$ al., 1988) was used in this study. Parasites were maintained by weekly passages in liver infusion tryptose medium (LIT) (Camargo, 1964); epimastigotes were transformed into metacyclic trypomastigotes in artificial triatomine urine medium (TAU) $(190 \mathrm{~mm}-\mathrm{NaCl}, 17 \mathrm{mM}-\mathrm{KCl}$, $2 \mathrm{~mm}-\mathrm{MgCl}_{2}, 2 \mathrm{~mm}-\mathrm{CaCl}_{2}, 8 \mathrm{~mm}$-potassium phosphate buffer, $\mathrm{pH} \mathrm{6.0)}$ supplemented with $10 \mathrm{mM}$-L-proline (TAUP) as previously described (Contreras et al., 1985).

Agglutination with lectins. Agglutination tests were done with a Takatsy microtitrator (Cooke Engineering Co.) using suspensions containing $2 \times 10^{8}$ cells $\mathrm{ml}^{-1}$, as described by Saraiva et al. (1986). The following lectins, purified as previously described (Pereira et al., 1980; Esteves et al., 1982), were used: Triticum vulgaris (WGA), Bandeiraea simplicifolia II (BS-II), Aaptus papillata (APL), Wistaria floribunda (WFH), Glycine max (SBA), Vicia villosa (VVL), Maclura pomifera (MPL), Bandeiraea simplicifolia I (BS-I), Arachis hypogaea (PNA), Ricinus communis I (RCA-I), Aximella polypoides (AxP), Artocarpus integrifolia (jacaline), Canavalia ensiformis (Con A) and Lens culinaris (LCL).

Radio-iodination of lectins. The lectins WGA, jacaline, PNA and BS-I were labelled with ${ }^{125}$ I as described by Saraiva et al. (1986). The minimum agglutination dose of each iodinated lectin was the same as that of the unlabelled lectin, as determined with the different development stages of $T$. cruzi.

Neuraminidase activity assay. This was done using the PNA haemagglutination method according to Pereira (1983a). Briefly, human $\mathrm{A}$ erythrocytes from a healthy donor were incubated at a final concentration of $5 \%(\mathrm{v} / \mathrm{v})$ with supernatants of cell-free LIT and TAUP for $60 \mathrm{~min}$ at $37^{\circ} \mathrm{C}$. The reaction was stopped by washing the erythrocytes three times in phosphate-buffered saline (PBS; $150 \mathrm{~mm}$ $\mathrm{NaCl}, 20 \mathrm{~mm}$-sodium phosphate buffer, $\mathrm{pH} 7.5$ ) containing $0.5 \mathrm{mg}$ BSA ml-1 (PBS-BSA). Erythrocytes were then resuspended in PBSBSA and titrated against PNA by agglutination assay.

\section{Results}

\section{Metacyclogenesis}

The in vitro metacyclogenesis of $T$. cruzi under chemically defined conditions consists of incubating replicating (LIT) epimastigotes in TAU for a $2 \mathrm{~h}$ period followed by incubating the parasites in TAUP, resulting in the transformation of epimastigotes into metacyclic trypomastigotes after $72 \mathrm{~h}$. Under these conditions, three distinct developmental stages of the parasite can be defined: epimastigotes (Ep-LIT), differentiating epimastigotes (Ep-TAU) and metacyclic trypomastigotes (Mt). Our previous results indicate that TAUP Mt display biological properties identical to those of triatomine Mt (Contreras et al., 1988).
Table 1. Agglutinating activity of lectins during metacyclogenesis of $T$. cruzi

Values given are means of three experiments.

\begin{tabular}{lccc}
\hline \hline & \multicolumn{2}{c}{$\begin{array}{c}\text { Minimum concentration for } \\
\text { agglutination }\left(\mu \mathrm{ml}^{-1}\right)\end{array}$} \\
\cline { 2 - 4 } & Ep-LIT & Ep-TAU & $\mathrm{Mt}$ \\
\hline D-GlcNAc-binding lectins & & & \\
WGA & $62 \cdot 5$ & $250 \cdot 0$ & $250 \cdot 0$ \\
BS-II & $63 \cdot 5$ & $127 \cdot 0$ & $127 \cdot 0$ \\
APL & $30 \cdot 8$ & $61 \cdot 6$ & $30 \cdot 8$ \\
D-GalNAc-binding lectins & & & \\
WFH & $92 \cdot 4$ & $46 \cdot 2$ & $23 \cdot 1$ \\
SBA & $0 \cdot 14$ & $0 \cdot 14$ & $0 \cdot 14$ \\
VVL & $>1300 \cdot 0^{*}$ & $>1300 \cdot 0^{*}$ & $325 \cdot 0$ \\
MPL & $96 \cdot 2$ & $>770 \cdot 0^{*}$ & $>770 \cdot 0^{*}$ \\
BS-I & $>2000 \cdot 0^{*}$ & $>1000 \cdot 0$ & $250 \cdot 0$ \\
D-Gal-binding lectins & \multicolumn{3}{c}{} \\
PNA & $156 \cdot 0$ & $39 \cdot 0$ & $39 \cdot 0$ \\
RCA I & $>1000 \cdot 0^{*}$ & $500 \cdot 0$ & $125 \cdot 0$ \\
AxP & 3.9 & 3.9 & $7 \cdot 8$ \\
Jacaline & $36 \cdot 6$ & $146 \cdot 5$ & $>586 \cdot 0^{*}$ \\
D-Man-binding lectins & & & \\
Con A & $0 \cdot 97$ & $0 \cdot 97$ & 0.97 \\
LCL & $500 \cdot 0$ & $250 \cdot 0$ & $250 \cdot 0$ \\
\hline \hline
\end{tabular}

* No agglutination seen at the maximum concentration used.

\section{Agglutination tests}

Agglutination of the different developmental stages of $T$. cruzi by lectins is shown in Table 1 . The binding reaction is considered to be most specific with cells that are agglutinated at the lowest lectin concentration. All types of agglutination were observed with all lectins: somaticsomatic (S-S), somatic-flagellum (S-F) and flagellumflagellum $(\mathrm{F}-\mathrm{F})$. All the developmental stages tested were unreactive with L-fucose-binding lectins, including those of Lotus tetragonolobus and Ulex europaeus.

D-GlcNAc-binding lectins. Ep-LIT, Ep-TAU and Mt were strongly agglutinated by APL. However, a decreased agglutination activity was observed during the metacyclogenesis process for BS-II and WGA. All these agglutinations were inhibited by $0 \cdot 1 \mathrm{M}-\mathrm{D}-\mathrm{GlcN}$ Ac.

D-GalNAc-binding lectins. SBA displayed a strong affinity for all developmental stages. With WFH the transformation of epimastigotes into metacyclic trypomastigotes was accompanied by increased cell interaction. However, the most striking differences were observed for VVL and BS-I, which selectively agglutinated Mt, and MPL, which preferentially agglutinated Ep-LIT. These agglutinations were inhibited by $0.1 \mathrm{M}-$ D-GalNAc.

$\mathrm{D}-$ Gal-binding lectins. The reactivities of PNA and AxP were strong for all developmental stages. However, 


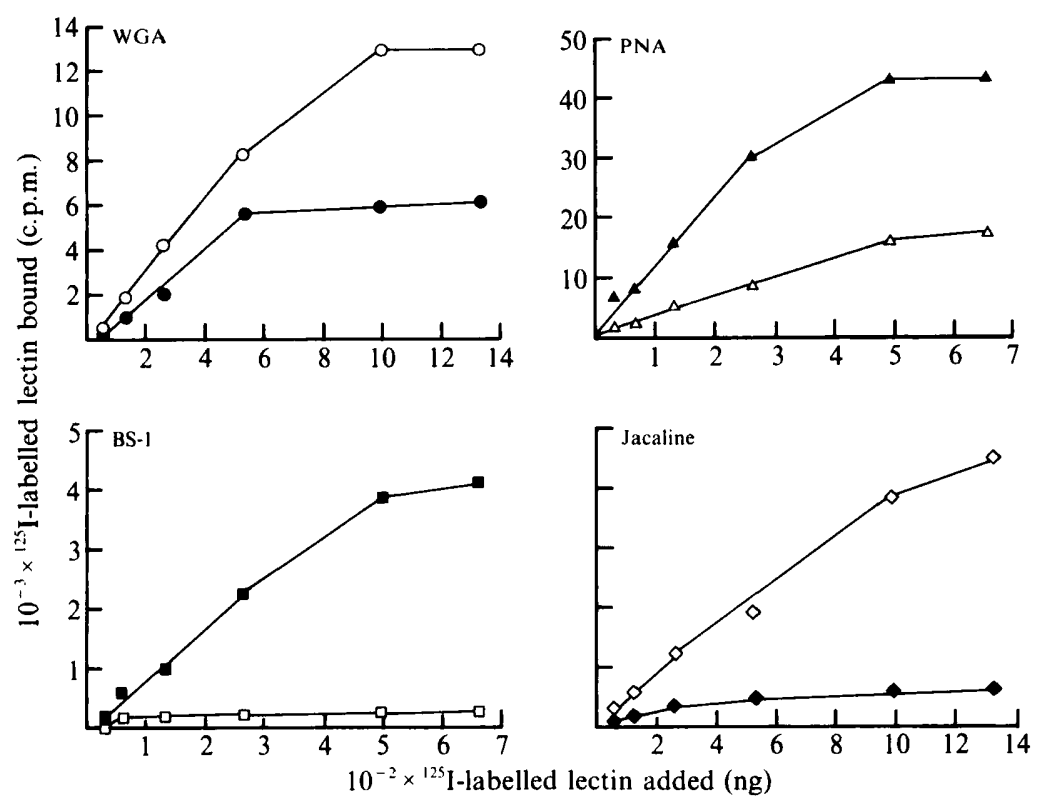

Fig. 1. Binding of ${ }^{125}$ I-labelled lectins to Ep-LIT (open symbols) and Mt (filled symbols) of T. cruzi Dm28c. Each point is the mean of three separate binding experiments, the results from which differed by no more than $10 \%$.

Table 2. Agglutination of human A erythrocytes in the presence of PNA after incubation with supernatants collected during metacyclogenesis of $T$. cruzi

Erythrocytes were in contact with $T$. cruzi culture supernatants for $60 \mathrm{~min}$ at $37^{\circ} \mathrm{C}$. Control erythrocytes did not agglutinate with PNA.

\begin{tabular}{lc}
\hline \hline Supernatant & $\begin{array}{c}\text { Minimum PNA concentration } \\
\text { required for agglutination }\left(\mu \mathrm{gl}^{-1}\right)\end{array}$ \\
\hline $\begin{array}{l}\text { LIT epimastigotes } \\
\text { TAUP metacyclic } \\
\text { trypomastigotes }\end{array}$ & $250 \cdot 0$ \\
\hline \hline
\end{tabular}

Table 3. Binding of ${ }^{125}$ I-labelled BS-I to Ep-LIT and ${ }^{125}$ I-labelled jacaline to Mt of T. cruzi

Values were calculated as in Fig. 2; only the major binding sites for each lectin were considered.

\begin{tabular}{|c|c|c|}
\hline Lectin & $\begin{array}{c}10^{-6} \times k \\
\text { (M) }\end{array}$ & $\begin{array}{c}10^{-5} \times \text { No. of } \\
\text { lectin sites } \\
\text { per cell }\end{array}$ \\
\hline Jacaline & 5.8 & $3 \cdot 3$ \\
\hline BS-I & 4.5 & 3.9 \\
\hline
\end{tabular}

differences were observed for jacaline and RCA-I: the former displayed a decreasing affinity and the latter an increasing affinity during the course of the metacyclogenesis process. The specificity of the agglutination reactions was confirmed by their inhibition with $0 \cdot 1 \mathrm{M}-\mathrm{D}$ Gal.
D-Man-binding lectins. Con A and LCL reacted with all developmental stages of $T$. cruzi. However, Con A showed a stronger affinity than LCL. These agglutinations were inhibited by $0 \cdot 1 \mathrm{M}-\alpha$-D-methyl mannoside.

\section{Neuraminidase activity}

Neuraminidase activity can be assayed by incubating human A erythrocytes with PNA, since these cells do not bind PNA unless they are first treated with neuraminidase (Pereira, 1983a; Libby et al., 1986). Incubating erythrocytes with LIT and TAUP cell-free supernatants resulted in their agglutination by PNA, indicating the presence of neuraminidase activities in the supernatants. This activity was more pronounced in TAUP supernatant (Table 2).

\section{Binding of ${ }^{125}$ I-labelled lectins}

In order to verify if the differences detected in the cell agglutination pattern with lectins (Table 1) were compatible with the number of the respective cell-surfaceexposed receptors, we tested the binding of ${ }^{125}$ I-labelled WGA, BS-I, PNA and jacaline to the different developmental forms of $T$. cruzi. This was necessary since the absence of agglutination by lectins does not necessarily mean absence of interaction with a particular cell (Nicolson, 1974). As clearly seen in Fig. $1(a-d)$, all four ${ }^{125}$ I-labelled lectins tested were able to discriminate EpLIT from Mt. The binding was specific since it could be reversed with a $0 \cdot 1 \mathrm{M}$ concentration of the corresponding 


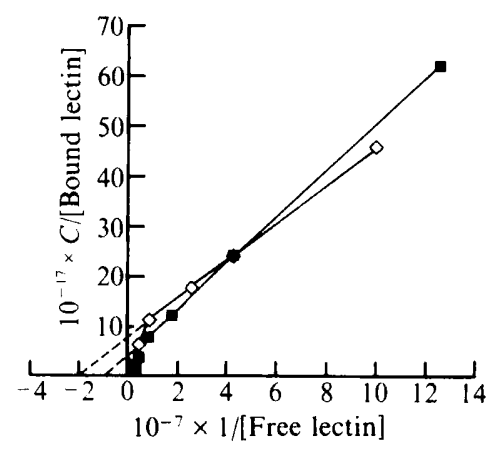

Fig. 2. Binding of $125 \mathrm{I}$-labelled jacaline to Ep-LIT $(\diamond)$ and ${ }^{125} \mathrm{I}-$ labelled BS-I to Mt ( $\square)$ of T. cruzi Dm28c. The data have been plotted by the method of Steck \& Wallach (1965) according to the equation

$$
\frac{C}{\text { [Bound lectin] }}=\frac{1}{K \cdot n} \cdot \frac{1}{[\text { Free lectin] }}+\frac{1}{n}
$$

where $C$ is the concentration of cells, $n$ is the number of lectin molecules bound per cell and $K$ is the association constant. For each lectin, only the high-affinity receptors were considered. All points represent means of triplicate experiments; the SD is less than $10 \%$.

specific inhibitor saccharide. The differential agglutination of the various developmental forms of $T$. cruzi by these four lectins (Table 1) correlates with the number of exposed ${ }^{125}$ I-labelled lectin receptor sites on each. The association constant and the mean numbers of lectin receptors per cell (Table 3) were calculated by plotting the binding data for BS-I and jacaline according to Steck \& Wallach (1965). BS-I interacted more strongly with Mt (Fig. 1) whereas the binding of jacaline was higher with Ep-LIT (Fig. 1). It should be noted that the interaction of both lectins is heterogeneous, but only their major receptor sites were considered (Fig. 2).

\section{Discussion}

The results presented above indicate that important changes in exposed surface carbohydrates occur during metacyclogenesis of $T$. cruzi, although the cell-surface saccharide moieties resembled those detected in other trypanosomatids (Pereira et al., 1980; Esteves et al., 1982, 1987, 1988; Saraiva et al., 1986). However, the pattern of lectin-binding sites obtained for $T$. cruzi Dm28c (isolated from opossum) differed from that obtained for the Y strain (isolated from man) (Pereira $e t$ al., 1980). This observation is in agreement with the generally described heterogeneity of the parasite (Morel et al., 1980; Dvorak et al., 1980; Engel et al., 1982; Schottelius \& Uhlenbruck, 1983; Tibayrenc et al., 1986; Stevens et al., 1988. Aymerich \& Goldenberg, 1989).

The differences observed in agglutination by lectins for the different developmental stages must reflect quite precisely the surface-carbohydrate composition of the parasite since the differentiation medium is chemically defined, containing only proline and glucose as carbon sources. This point is particularly relevant for the surface-sugar composition of trypomastigote forms, which in other studies have been obtained from infected mice and from tissue culture (Pereira et al., 1980). Transformation of epimastigotes into metacyclic trypomastigotes was followed by acquisition of cell-surfacespecific receptors for D-GalNAc- and D-Gal-lectins (VVL and RCA-I, respectively). Hence, cells of $T$. cruzi appear to synthesize rather than take up available carbohydrates from the environment.

Significant differences in agglutination by lectins were observed after incubation of Ep-LIT for only $2 \mathrm{~h}$ in TAU, for example increased agglutinating activity of PNA and RCA-I and decreased agglutination with WGA and MPL (Table 1). For some lectins, Ep-TAU resembled Mt much more than Ep-LIT, suggesting that changes in the surface sugar composition precede the morphological transformation of epimastigotes into metacyclic trypomastigotes. This is in agreement with previous observations concerning the fatty acid composition of differentiating epimastigotes (Esteves et al., 1989).

Neuraminidase activity could be detected in cell culture supernatants (Table 2). Detection of neuraminidase in TAU suggests that it is secreted by the parasite, which could partially explain the low reactivity of WGA with Ep-TAU, since WGA reacts with $N$-acetylneuraminic acid as well as D-GlcNAc. Furthermore, this finding could also explain the increased binding of PNA (specific for D-Gal) since neuraminidase action leads to the generation of new galactose-receptor sites (Pereira, $1983 a$ ). Several studies indicate that neuraminidase may be involved in the infection of vertebrate host cells and in the release of trypomastigotes from these cells (Pereira, $1983 b$ ).

Studies with ${ }^{125}$ I-labelled WGA, PNA, jacaline and BS-I (Fig. 1) indicate that the agglutination induced by these lectins is clearly dependent on the number and type of cell-surface lectin receptors, and not on other factors such as the mobility and accessibility of the receptors. In addition, these studies allowed the determination of the density of receptor sites during metacyclogenesis of $T$. cruzi. The number of jacaline (on Ep-LIT) and BS-I (on $\mathrm{Mt}$ ) receptor sites were $3.3 \times 10^{5}$ and $3.9 \times 10^{5}$ per cell, respectively: these values fall well within the range of the number of cell-surface lectin-receptor sites $\left(10^{5}-10^{6}\right)$ detected for other trypanosomatids (Pereira et al., 1980; Esteves et al., 1982; Saraiva et al., 1986).

The observed changes in surface-exposed carbohydrates during metacyclogenesis of $T$. cruzi under chemically defined conditions, as judged by lectin-binding 
studies, might ultimately be related to the differentiation process occurring inside the triatomine intermediary host. It is possible that the interaction of cell-surface glycoproteins with lectins, which have been described in the gut of the insect vector (Pereira et al., 1981), might play a role in the morphogenesis of $T$. cruzi. Clearly, further biochemical characterization of the glycoconjugates on the surface of $T$. cruzi will be needed to help clarify their possible role during differentiation of the parasite.

We thank Catherine Lowndes for the critical reading of this manuscript, Armindo Caldeira for technical assistance and the Setor de Multi-Meios (Fiocruz) for the drawings and photographs. This work received financial support from UNDP/WORLD BANK/WHO Special Programme for Research and Training in Tropical Diseases, Conselho Nacional de Desenvolvimento Cientifico e Tecnológico and CPEG-UFRJ.

\section{References}

AYMeRICH, S. \& GoldenberG, S. (1989). The karyotype of Trypanosoma cruzi $\mathrm{Dm} 28 \mathrm{c}$ : comparison with other $T$. cruzi strains and trypanosomatids. Experimental Parasitology 69, 107-115.

Bonaldo, M. C., Souto-Padron, T., DE Souza, W. \& Goldenberg, S. (1988). Cell-substrate adhesion during Trypanosoma cruzi differentiation. Journal of Cell Biology 106, 1349-1358.

Brener, Z. (1973). Biology of Trypanosoma cruzi. Annual Review of Microbiology 27, 347-383.

Camargo, E. P. (1964). Growth and differentiation of Trypanosomo cruzi. I. Origin of metacyclic trypomastigotes in liquid media. Revista do Instituto de Medicina Tropical de Sao Paulo 6, 93-100.

Contreras, V. T., Salles, J. M., Thomas, N., Morel, C. M. \& GOLDENBERG, S. (1985). In vitro differentiation of Trypanosoma cruzi under chemically defined conditions. Molecular and Biochemical Parasitology 16, 315-327.

Contreras, V. T., Araujo-Jorge, T. C., Bonaldo, M. C., Thomas, N., Barbosa, H. S., Meirelles, M. N. L. \& GoldenberG, S. (1988). Biological aspects of the Dm 28c clone of Trypanosoma cruzi after metacyclogenesis in chemically defined media. Memorias do Instituto Oswaldo Cruz 83, 123-133.

Dvorak, J. A., Hall, T. E., Crane, M. S. J., Engel, J. C., MCDaniel, J. P. \& URIEGAS, R. (1980). Trypanosoma cruzi; flow cytometric analysis. I. Analysis of total DNA per organism by means of mithramycin-induced fluorescence. Journal of Protozoology 29, 430437.

Engel, J. C., Dvorak, J. E., Segura, E. L. \& Crane, M. S. J. (1982). Trypanosoma cruzi: biological characterization of 19 clones derived from two chronic chagasic patients. I. Growth kinetics in liquid medium. Journal of Protozoology 29, 555-560.
Esteves, M. G., Andrade, A. F. B., Angluster, J., de Souza, W., Mundim, M. H., Roitman, I. \& Pereira, M. E. A. (1982), Cell surface carbohydrates in Crithidia deanei: influence of the endosymbiont. European Journal of Cell Biology 26, 244-248.

Esteves, M. G., Andrade, A. F. B., Alviano, C. S., Roitman, I., De SouzA, W. \& ANGluster, J. (1987). Cell surface carbohydrate differences in wild and mutant strains of Crithidia fasciculata. Journal of Protozoology 34, 226-230.

Esteves, M. G., Attias, M., Silva-Filho, F. C., Pereira, M. E. A., Alviano, C., ANGluster, J. \& de SouzA, W. (1988). The cell surface of Phytomonas davidi. Cytobiosis 54, 71-84.

Esteves, M. G., Gonzales-Perdomo, M., Alviano, C. S., AnglusTER, J. \& GOLDENBERG, S. (1989). Changes in fatty-acid composition associated with differentiation of Trypanosoma cruzi. FEMS Microbiology Letters 59, 31-34.

Libby, P., Alroy, J. \& Pereira, M. E. A. (1986). A neuraminidase from Trypanosoma cruzi removes sialic acid from the surface of mammalian myocardial and endothelial cells. Journal of Clinical Investigation 77, 127-135.

Morel, C. M., Chiari, E., Camargo, E. P., Mattei, D. M., Romanha, A. J. \& Simpson, L. (1980). Strains and clones of Trypanosoma cruzi can be characterized by pattern of restriction endonuclease products of kinetoplast DNA minicircles. Proceedings of the National Academy of Sciences of the United States of America 77, 6810-6814.

Nicolson, G. L. (1974). The interaction of lectins with animal cell surfaces. International Review of Cytology 39, 89-190.

Pereira, M. E. A. $(1983 a)$. A rapid and sensitive assay for neuraminidase using peanut lectin hemagglutination: Application to Vibrio cholerae and Trypanosoma cruzi. Journal of Immunological Methods 63, 25-34.

Pereira, M. E. A. (1983b). A developmentally regulated neuraminidase activity in Trypanosoma cruzi. Science 219, 1444-1446.

Pereira, M. E. A., Loures, M. A., Villalta, F. \& Andrade, A. F. B. (1980). Lectin receptors as markers for Trypanosoma cruzi developmental stages and a study of the interaction of wheat germ agglutinin with sialic acid residues on epimastigote cells. Journal of Experimental Medicine 152, 1375-1392.

Pereira, M. E. A., Andrade, A. F. B. \& Ribeiro, J. M. C. (1981). Lectins of distinct specificity in Rhodnius prolixus interact selectively with Trypanosoma cruzi. Sciences 211, 597-600.

Saraiva, E. M. B., Andrade, A. F. B. \& Pereira, M. E. A. (1986). Cell surface carbohydrate of Leishmania mexicana amazonensis: differences between infective and non-infective forms. European Journal of Cell Biology 40, 219-225.

SChottelius, J. \& Uhlenbruck, G. (1983) Comparative studies of Trypanosoma cruzi and $T$. cruzi like stocks from different South American countries using lectins. Zeitschrift für Parasitenkunde 69, 727-736.

STECK, T. L. \& WALLACH, D. F. H. (1965). The binding of kidney-bean phytohemagglutinin by Ehrlich ascites carcinoma. Biochimica et Biophysica Acta 97, 510-522.

Stevens, A. F., Miles, M. A. \& Allen, A. K. (1988) Trypanosoma cruzi: studies on the interactions of lectins with glycoconjugates of different zymodemes. Experimental Parasitology 67, 324-333.

Tibayrenc, M., Ward, P., Moya, A. \& Ayalla, F. J. (1986). Natural populations of Trypanosoma cruzi, the agent of Chagas disease, have a complex multiclonal structure. Proceedings of the National Academy of Sciences of the United States of America 83, 115-119. 